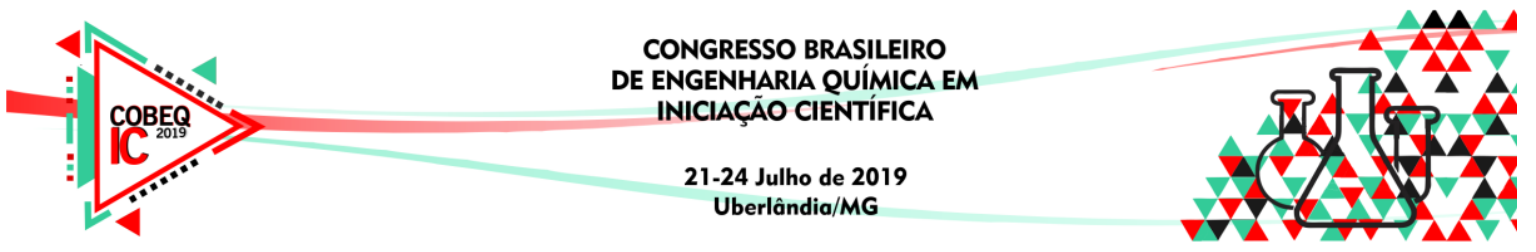

\title{
ESTUDO NUMÉRICO DO FENÔMENO DE SEGREGAÇÃO DE MATERIAL GRANULAR EM TAMBOR ROTATÓRIO
}

\author{
P. F. SILVA ${ }^{1}$, R. J. BRANDÃO ${ }^{1}$, C. R. DUARTE ${ }^{1}$ e M. A. S. BARROZO ${ }^{1}$ \\ ${ }^{1}$ Universidade Federal de Uberlândia, Faculdade de Engenharia Química \\ E-mail para contato: patrikmotafs@hotmail.com
}

\begin{abstract}
RESUMO - Um dos motivos da utilização de tambores rotatórios em processos industriais deve-se a sua geometria relativamente simples, que consiste em uma estrutura cilíndrica horizontal, e que gira em torno do seu próprio eixo. Dentre as problemáticas apresentadas na utilização do tambor rotatório, a segregação de partículas, aparece como expoente. Deste modo este trabalho tem como objetivo estudar o fenômeno de segregação e mistura por meio de técnicas numéricas. No estudo numérico foi utilizada a abordagem numérica Lagrangeana, através do Método dos Elementos Discretos (DEM), onde cada partícula é acompanhada, individualmente, com a consequente aplicação do balanço de forças, levando em consideração as interações com as demais partículas através de leis de contato. Foi estudado o fenômeno de segregação em tambor rotatório, avaliando a influência do tamanho e densidade de partículas. Tal estudo permitiu analisar e compreender o efeito das variáveis no processo. A densidade é diretamente proporcional ao índice de segregação, enquanto o tamanho apresentou um ponto de máximo.
\end{abstract}

\section{INTRODUÇÃO}

Um tambor rotatório é um cilindro que gira em torno do seu eixo axial e geralmente é operado inclinado na horizontal. Este equipamento tem papel primordial quando se refere a escoamento de material granular. Devido a sua construção simples, alta capacidade de operação e facilidade de limpeza, os tambores são empregados em diversas operações unitárias como: mistura, granulação, moagem, secagem e em processos químicos. (SANTOMASO,2003)

No entanto, a segregação é um dos problemas que se manifesta quando se faz uso do tambor rotatório e esse fenômeno causa uma desconformidade na mistura granular, o que reduz a qualidade de produtos cuja homogeneização é relevante, como por exemplo na produção de fertilizantes e fármacos. (KUO et al., 2005)

Estudos já realizados concluíram que quando partículas de diferentes propriedades (tamanho, densidade, formato, etc) são processadas, o efeito da segregação é pronunciado (KUO et al., 2005; PIRKER et al., 2010; SANTOS et al., 2013). 


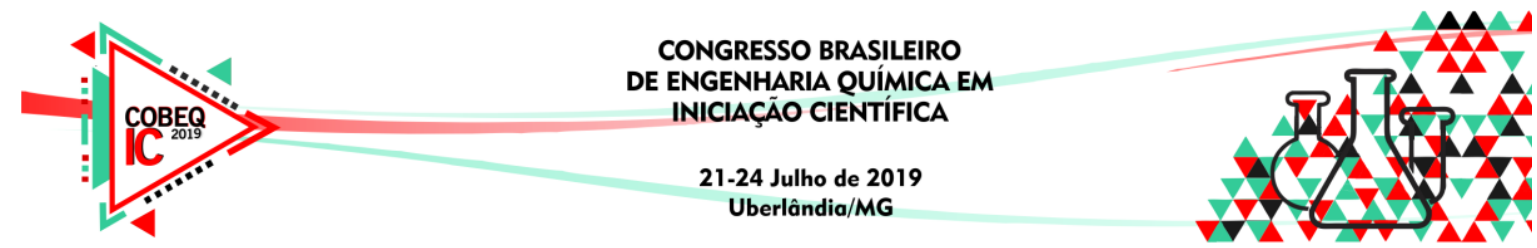

Desta maneira torna-se necessário o estudo da dinâmica de partículas no interior de tambores rotatórios e do fenômeno de segregação, avaliando o efeito da variação das características do material, como tamanho e densidade sobre o fenômeno de segregação.

\section{MATERIAIS E MÉTODOS}

Para o estudo numérico escolheu-se partículas com diâmetros na faixa de 0,6 a 6,0 mm e densidade de 1000 a $10000 \mathrm{~kg} / \mathrm{m}^{3}$ para serem simuladas no tambor rotativo. Foi selecionado para o estudo um grau de preenchimento de $25 \%$.

Para compor uma mistura que fosse adequada para as análises, foi adotado diferentes razões (variando de 0,1 a 0,9) de tamanho e densidade a fim de compreender melhor o efeito da diferença do tamanho e densidade das partículas na dinâmica de segregação, sendo estas dispostas lado a lado no interior do tambor.

\subsection{Configurações Da Simulação}

Todas as simulações foram conduzidas no software livre LIGGGHTS, utilizando o modelo de contato de Hertz-Mindlin. Para garantir a estabilidade numérica, utilizou-se um passo no tempo equivalente a 5\% do passo de tempo de Rayleigh e o tempo simulado foi de 50 segundos para todas as simulações. As dimensões do leito simulado foram de $30 \mathrm{~cm}$ de diâmetro e $50 \mathrm{~cm}$ de comprimento. A Tabela 1 apresenta de forma simplificada todas as condições utilizadas para a realização do estudo numérico.

Tabela 1 - Condições utilizadas para simulação Lagrangeana

\begin{tabular}{|c|c|}
\hline Modelo & Hertz-Mindlin \\
\hline Passo no Tempo (s) & $2 \times 10-5$ \\
\hline Tempo de Simulação (s) & 50 \\
\hline Tamanho de Célula & 3 Rmin \\
\hline \multirow{2}{*}{ Razão de Poisson [-] } & Partícula 0,25 \\
\hline & Parede 0,30 \\
\hline \multirow{2}{*}{ Módulo de Cisalhamento (Pa) } & Partícula $2,61 \times 10^{7}$ \\
\hline & Parede $7,00 \times 10^{10}$ \\
\hline
\end{tabular}

\subsection{Medida Do Índice De Segregação}

Imagens do leito simulado foram extraídas, em tempos predeterminados, para análise da cinética de segregação. A secção transversal preenchida do tambor simulado foi dividida em 20 células (Figura 1), e então foram contabilizadas as concentrações volumétricas de cada material. 


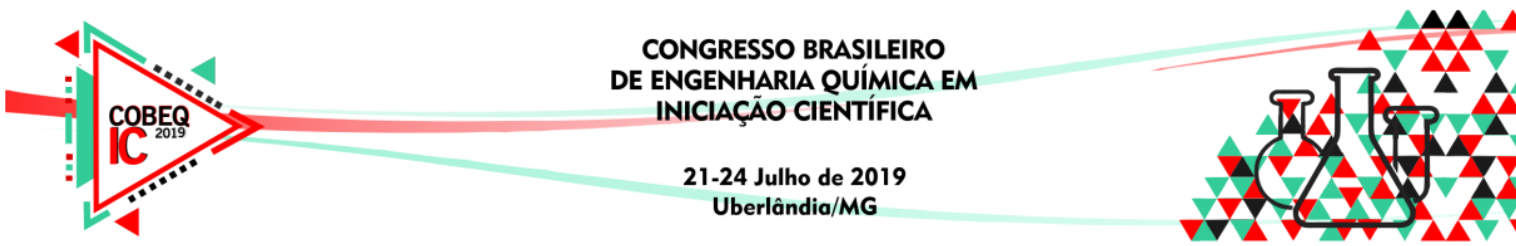

Figura 1 - Gradeamento transversal para determinação do índice de segregação.

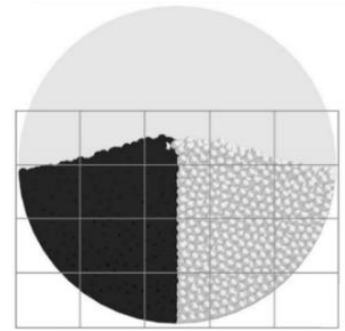

Foram então calculados os índices de segregação $(\sigma)$, o qual é definido como o desvio padrão das concentrações (Equação 1), permitindo a realização de uma análise quantitativa.

$$
\sigma=\sqrt{\frac{\sum_{i=1}^{N}\left(C-C_{\text {médio }}\right)^{2}}{N-1}}
$$

Sendo C a concentração volumétrica local, $C_{(m e ́ d i o)}$ a concentração volumétrica média, e $\mathrm{N}$ o número total de células ocupadas pelas partículas, respectivamente. Os valores do índice de segregação variam de 0 , no caso em que as partículas se encontram perfeitamente misturadas, e 0,5, no caso de uma completa segregação (SANTOS, 2015).

Os índices de segregação foram determinados e as cinéticas de segregação elaboradas para cada umas das combinações de razão de tamanho e de densidade.

\section{RESULTADOS E DISCUSSÕES}

\subsection{Efeito Da Densidade Da Partícula}

Na Figura 2 é apresentado o comportamento do índice de segregação em função da razão de densidade.

Figura 2 - Efeito da razão de densidade no índice de segregação.

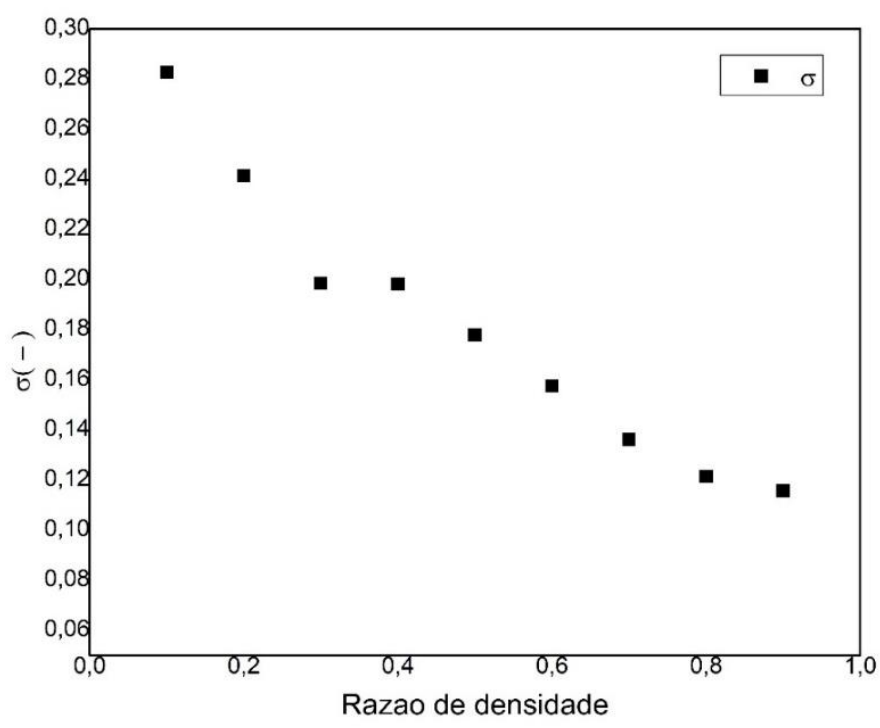




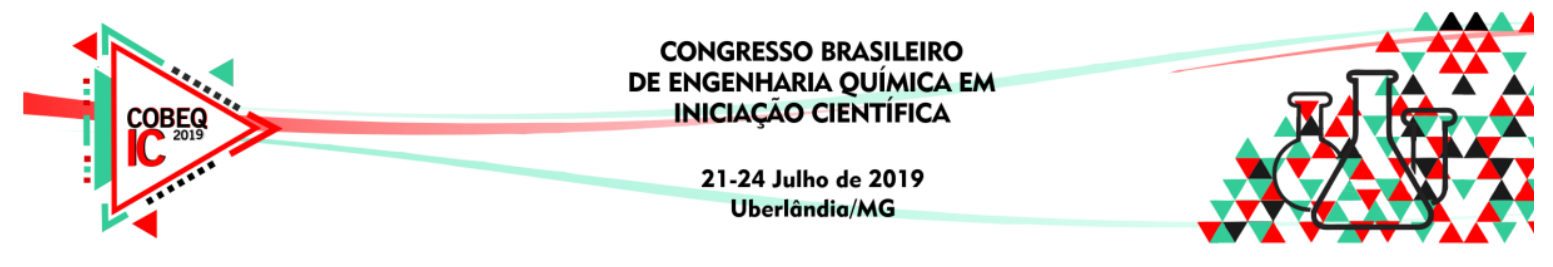

Na Figura 3 é apresentado o comportamento do índice de segregação em função do tempo para as diferentes razões de densidade.

Figura 3 - Índice de segregação em função do tempo para razões de densidade diferentes.

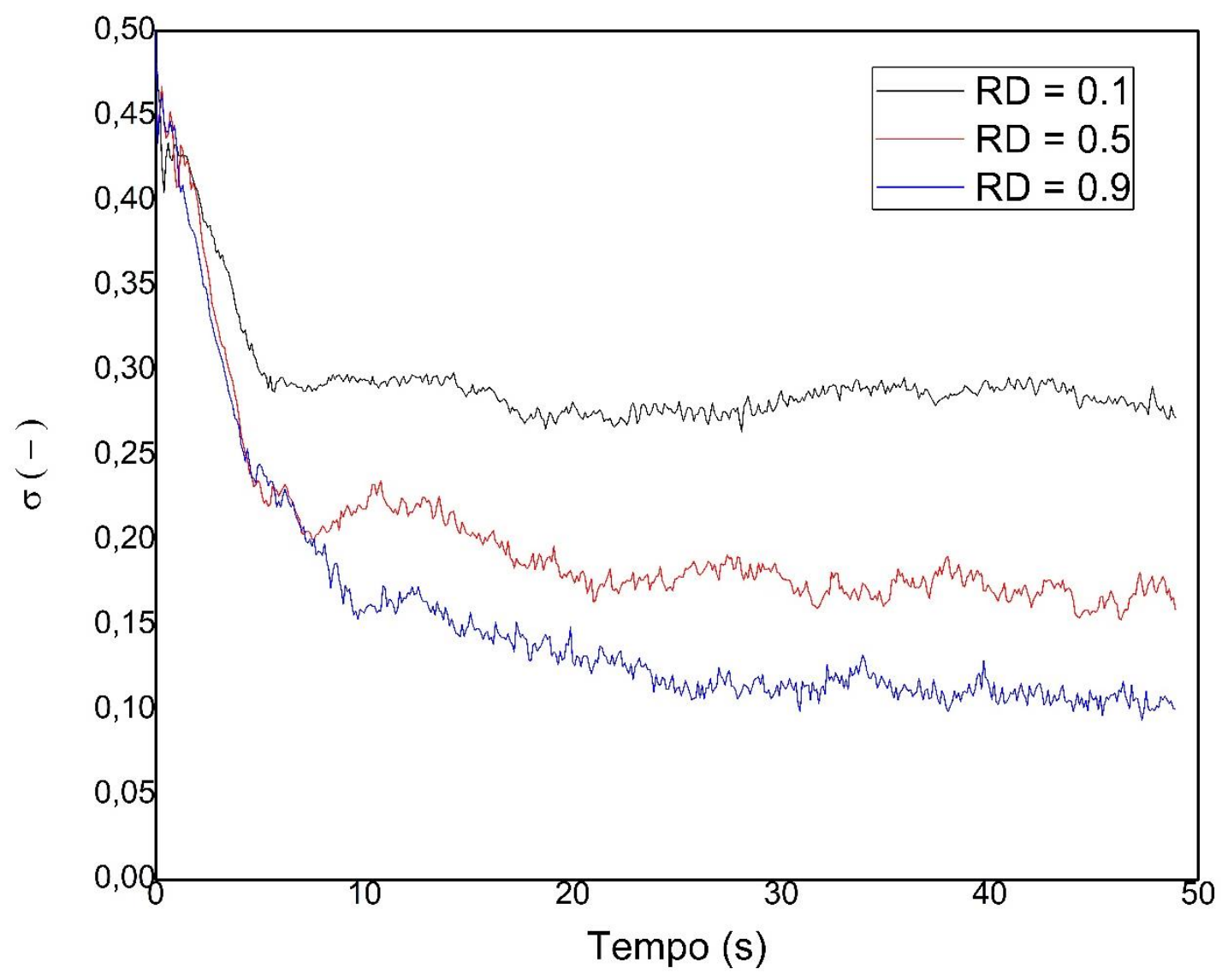

De acordo com as Figuras 2 e 3 nota-se que o índice de segregação é mais pronunciado quando a diferença de densidades é maior. Estudos já realizados (Yamamoto 2015; Chen et al 2016.) mostraram que as partículas mais densas tenderam a ficar localizadas próximo do eixo central do leito, enquanto as partículas menos densas segregavam-se radialmente à periferia.

Por causa do modo como o tambor opera no regime de rolamento, as partículas que se encontram na parte inferior do tambor se movem até uma altura máxima e depois escoam pelo leito. Como resultado, as partículas mais densas penetram e permanecem no centro da seção transversal do tambor. Segundo Chen et al. (2016), este fenômeno pode ser atribuído ao mecanismo de condensação, no qual partículas mais densas migram para parte inferior do fluxo granular.

\subsection{Efeito Do Tamanho Da Partícula}

Na Figura 4 é apresentado o comportamento do índice de segregação em função da razão de tamanho. 
Figura 4 - Efeito da razão de tamanho no índice de segregação.

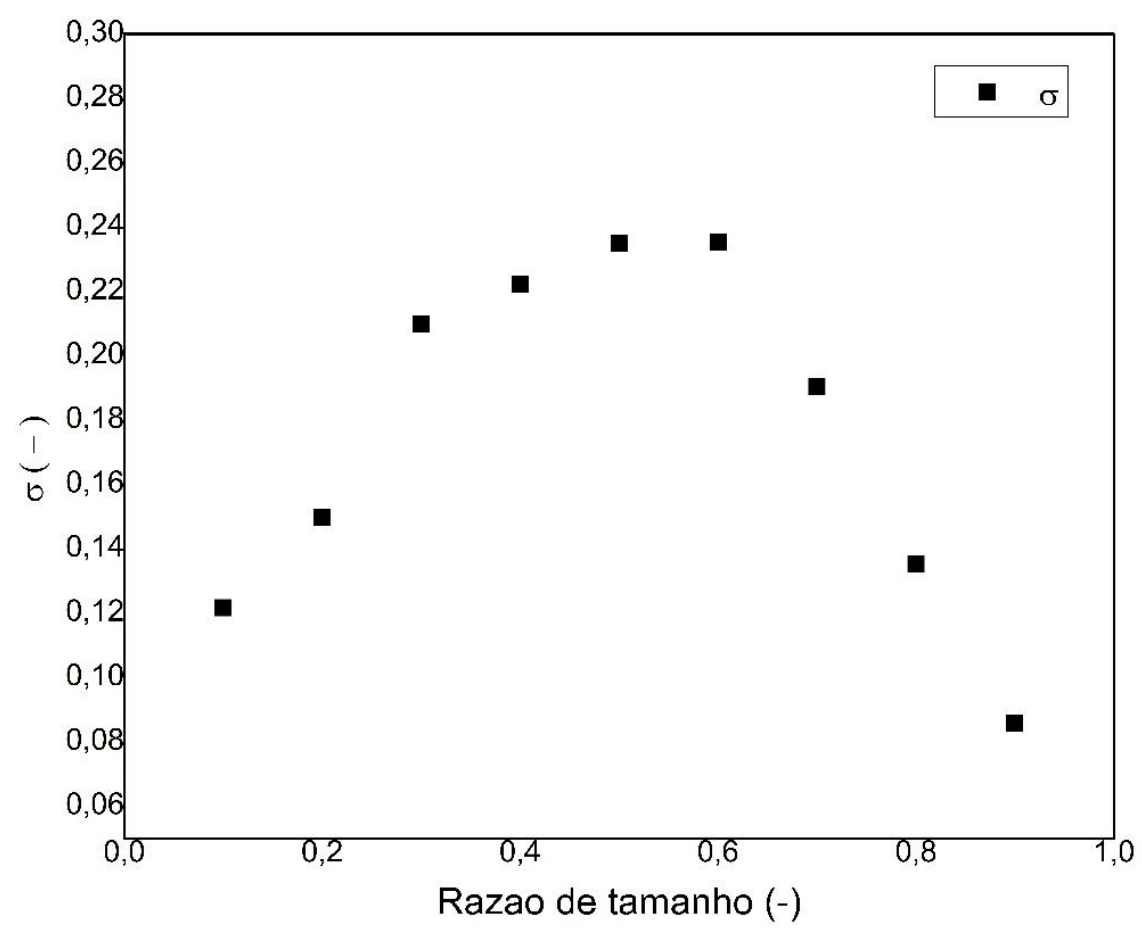

Na Figura 5 é apresentado o comportamento do índice de segregação em função do tempo para as diferentes razões de tamanho.

Figura 5 - Índice de segregação em função do tempo para razões de tamanhos diferentes.

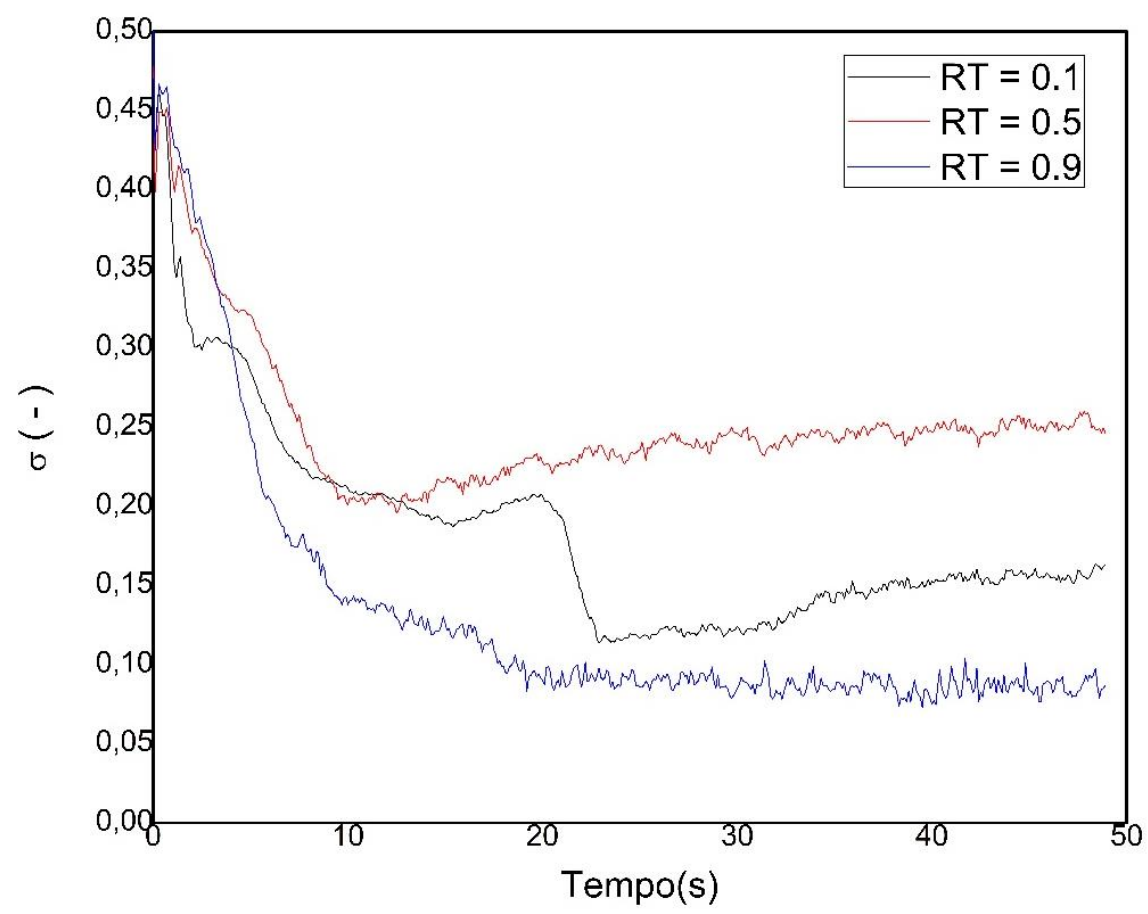




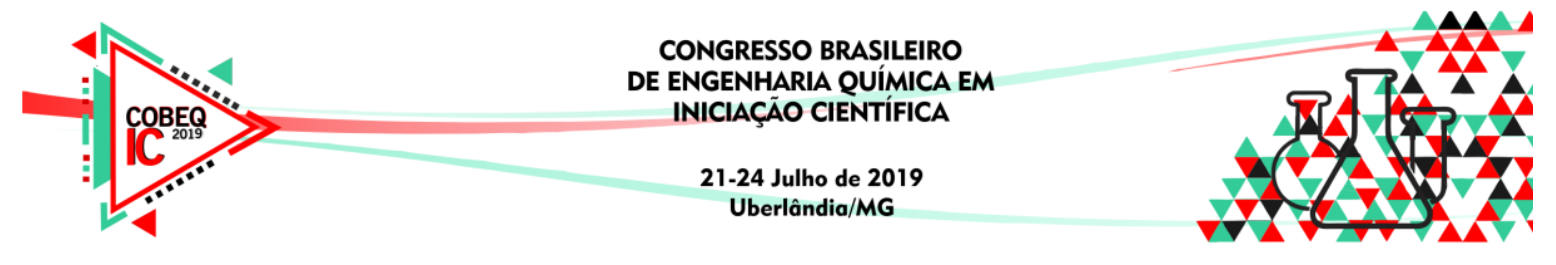

De acordo com a Figura 4 nota-se que existe uma razão de tamanhos para a qual o índice de segregação é máximo. Conforme a razão de tamanhos tende a 1 (um) o tamanho das partículas se aproxima e o efeito da segregação é pouco pronunciado. No entanto, quando a razão de tamanho diminui, a diferença de dimensão entre partículas aumenta, fazendo com que os grânulos de menor diâmetro migrem para o núcleo radial através do mecanismo de percolação, intensificando a segregação. Entretanto, nota-se que ao diminuir a razão de diâmetro das partículas para valores inferiores a 0,5 , um comportamento de redução do índice de segregação é evidenciado (Figuras 4 e 5), isto ocorre porque, partículas pequenas, com dimensões equivalentes aos interstícios do leito formado por partículas maiores, conseguem percolar reduzindo a formação do núcleo radial e consequentemente o fenômeno de segregação.

\section{CONCLUSÃO}

A partir dos resultados obtidos, conclui-se que tanto a razão de tamanhos quanto a razão de densidades dos materiais influenciam o fenômeno de segregação.

Foi mostrado que quanto menor a razão de densidades das partículas mais pronunciado é o efeito de segregação. Enquanto que para a razão de tamanhos existe um valor máximo para o índice de segregação.

Para ambos efeitos analisados, razões de tamanho e densidade próximos de um maximizam a eficiência de mistura do sistema.

\section{REFERÊNCIAS}

CHEN, H.; ZHAO, X. Q.; XIAO, Y. G.; LIU, Y. L.; LIU, Y. Radial mixing and segregation of granular bed bi-dispersed both in particle size and density within horizontal rotating drum. Transactions of Nonferrous Metals Society of China (English Edition), v. 26, n. 2, p. 527- 535, 2016.

KUO, H. P.; HSU, R. C.; HSIAO, Y. C. Investigation of axial segregation in a rotating drum. Powder Technology, v. 153, n. 3, p. 196-203, 2005.

PIRKER, S.; KAHRIMANOVIC, D.; KLOSS, C.; POPOFF, B.; BRAUN, M. Simulating coarse particle conveying by a set of Eulerian, Lagrangian and hybrid particle models. Powder Technology, v. 204, n. 2-3, p. 203-213, 2010.

SANTOMASO, A. C.; DING, Y. L.; LICKISS, J. R.; YORK, D. W. Investigation of the Granular Behaviour in a Rotating Drum Operated over a Wide Range of Rotational Speed. Chemical Engineering Research and Design, v. 81, n. 8, p. 936-945, 2003.

SANTOS, D. A.; DADALTO, F. O.; SCATENA, R.; DUARTE, C. R.; BARROZO, M. A. S. A hydrodynamic analysis of a rotating drum operating in the rolling regime. Chemical Engineering Research and Design, v. 94, n. August, p. 204-212, 2015.

SANTOS, D. A.; PETRI, I. J.; DUARTE, C. R.; BARROZO, M. A. S. Experimental and CFD study of the hydrodynamic behavior in a rotating drum. Powder Technology, v. 250, p. 52-62, 2013.

YAMAMOTO, M.; KANO, J.; ISHIHARA, S. Evaluation of particle density effect for mixing behavior in a rotating drum mixer by DEM simulation,Advanced Powder Technology, v. 27,p. 864-870, 2016. 